6. - A characterization of the Cayley numbers, Math. Assoc. America Studies in Mathematics, Vol. 2, pp. 126-143, Prentice-Hall, Englewood Cliffs, N. J., 1963.

Syracuse UNIVERSITY

\title{
A CONDITION FOR A FINITE GROUP TO BE NILPOTENT
}

\section{STEPHEN MONTAGUE AND GOMER THOMAS}

Let $\mathcal{H C}$ be a class of groups such that:

(i) If $G$ is in $\Re$, then every homomorphic image of $G$ is in $\Re$.

(ii) If $G$ is finite and $G / \phi(G)$ is in $\mathcal{H}$, where $\phi(G)$ is the Frattini subgroup of $G$, then $G$ is in $\mathfrak{H C}$.

Examples of such classes are the class of nilpotent groups and the class of supersolvable groups. Others can be found in a paper by Baer [1].

In this note a theorem of $\mathrm{P}$. Hall on nilpotent groups is proved as a corollary to the following:

THEOREM. If $G$ is a finite group with a subgroup $H$ such that $\phi(H)$ is normal in $G$ and $G / \phi(H)$ is in $\mathfrak{H C}$, then $G$ is in $\mathfrak{H C}$.

LEMMA (HUPPERT). Let $G$ be a finite group, $H$ be a subgroup of $G$, and $N$ be a subgroup of $H$ such that $N$ is normal in $G$ and $N \leqq \phi(H)$. Then $N \leqq \phi(G)$.

Proof. If not, $G$ would have to have a maximal subgroup $U$ such that $N \ddagger U$. Then $H=G \cap H=N U \cap H=N(U \cap H)=U \cap H$, since $N \leqq \phi(H)$. But this implies $H \leqq U$, contrary to $N \leqq U$.

Proof OF THEOREM. An application of the Lemma with $N=\phi(H)$ shows that $\phi(H) \leqq \phi(G)$. Hence $G / \phi(G)$ is in $\mathcal{F}$, and so $G$ is in $\mathcal{H}$.

CoRollary. If $G$ is a finite group with a normal subgroup $H$ such that $H$ is nilpotent and $G / H^{\prime}$ is nilpotent, where $H^{\prime}$ is the commutator subgroup of $H$, then $G$ is nilpotent.

Proof. Since $H$ is nilpotent, $\phi(H)$ contains $H^{\prime}$. Hence $G / \phi(H)$ is

Received by the editors September 22, 1965. 
nilpotent, and the theorem applies, with $\mathfrak{F}$ being the class of nilpotent groups.

This last result was originally proved by $\mathrm{P}$. Hall [2]. His proof gives an upper bound for the nilpotency class of $G$ in terms of those of $H$ and $G / H^{\prime}$, and it does not require finiteness of $G$, but it is fairly complicated.

The authors wish to thank Dr. Michio Suzuki for his advice and encouragement.

\section{BIBLIOGRAPHY}

1. R. Baer, Classes of finite groups and their properties, Illinois J. Math. 1 (1957), 115-187.

2. P. Hall, Some sufficient conditions for a group to be nilpotent, Illinois J. Math. 2 (1958), 787-801.

3. B. Huppert, Lecture notes, University of Illinois, Urbana, 1964.

UNIVERSITY OF ILLINOIS 